CERN-TH.7311/94

\title{
HARD PHOTON BREMSSTRAHLUNG EFFECTS IN HYPERON SEMILEPTONIC DECAYS
}

\author{
F. Glück* and I. Joó**
}

\begin{abstract}
An efficient Monte Carlo method for photon bremsstrahlung calculations in semileptonic decays is described. This method is useful for order- $\alpha$ radiative correction calculations, and for event generations in off-line Monte Carlo simulation programs. The order- $\alpha$ bremsstrahlung amplitude in hyperon semileptonic decays is given by using the Low-theorem, and the bremsstrahlung amplitude squared is calculated for unpolarized decays. Numerical calculation results are presented for $n \rightarrow p e \bar{\nu}, \mu \rightarrow e \bar{\nu}_{e} \nu_{\mu}, \Sigma^{-} \rightarrow n e \bar{\nu}$ and $\Lambda \rightarrow p e \bar{\nu}$ decays. The general features of the Monte Carlo method might be useful for photon bremsstrahlung calculations in other decay and collision processes.
\end{abstract}

To be submitted to Nuclear Physics B

${ }^{*}$ Research Institute for Nuclear and Particle Physics, H-1525 Budapest 114, P.O.B. 49, HUNGARY

** Department of Analysis, L. Eötvös University, H-1088 Budapest, Múzeum krt. 6-8, HUNGARY

CERN-TH.7311/94

July 1994 


\section{Introduction}

The large number of events detected in hyperon semileptonic decay experiments performed in the eighties [ $1-4]$ makes possible the precise determination of the free theoretical parameters of these decay processes. In addition to the small statistical and systematic errors of the experiments, the theoretical uncertainties are also required to be as small as possible, in order to exploit the possibilities of the precise experiments. Thus precise and reliable calculations of the radiative corrections to various measurable quantities are needed.

Many order- $\alpha$ electromagnetic correction calculations for hyperon semileptonic decays have been published in the last two decades [5-20]. Three of these papers use ultraviolet cutoff $[5,6]$ or dipole form factors [8] in order to handle the ultraviolet divergence problem. All the other papers employ the model dependent - model independent separation introduced by Sirlin [21] for neutron and nuclear $\beta$-decays ( this separation is explained for hyperon semileptonic decays in refs. $7,12,16$; see also sect. 1 in ref. 18 ). The bremsstrahlung part of the radiative correction is, from the theoretical point of view, much more easier to handle than the virtual correction. The energy of the bremsstrahlung photons in the semileptonic decays of the $\mathrm{SU}(3)$-octet baryons is small compared to the baryon masses, therefore it is a good approximation to assume that the bremsstrahlung photon is coupled minimally to the pointlike baryons $[5-12,14-16,18]$. The Low-theorem [22-27] guarantees that the bremsstrahlung matrix element can be more precisely determined, including not only the terms of order $K^{-1}$ but also the terms of order $K^{0}$ ( $K$ denotes the bremsstrahlung photon energy ) $[13,17,19,20]$. The terms of order $K$ in the bremsstrahlung amplitude depend on the details of the strong interaction, but they are small compared to the terms of order $K^{-1}$ and $K^{0}$, therefore they can be neglected.

There are two types of technical difficulties during the photon bremsstrahlung calculation. The first is the evaluation of the bremsstrahlung matrix element squared, and the second is the integration of this matrix element squared over the bremsstrahlung phase space. The bremsstrahlung matrix element squared calculations have been performed either "by hand" ( i.e.: on paper, without computer) $[5-11,13,15,17,19,20]$, or by the REDUCE symbolic algebraic software $[12,14,16,18]$. The bremsstrahlung integrations have been made analytically [5-11,13,15,17,19,20], numerically [12], or semianalytically ( by some combination of analytical and numerical methods) $[14,16,18]$. The analytical and semianalytical methods require the calculation of many complicated analytical integrals. On the other hand, the pure numerical method requires much computer time [28]. The different types of quantities in these methods need completely different calculations, and it is very hard to take into account the experimental details.

We describe in this paper a Monte Carlo method of order- $\alpha$ radiative correction calculations for hyperon semileptonic decays. This method has several advantages compared to the calculations published so far: 
1. With the aid of our method one can easily compute radiative corrections to every quantity or distribution. The hard bremsstrahlung photon effects can simply be calculated.

2. The formulae presented here are rather simple and easily verifiable.

3. This method is especially suitable for the experimental analyses, where the various kinematic cuts, detection efficiencies, energy resolution etc. necessitate to modify the theoretical distributions during the comparison of theory and experiment, in order to determine the free theoretical parameters as precisely as possible.

4. Our method is useful not only for radiative correction calculations, but also for event generation in the off-line Monte Carlo simulation programs.

The plan of the paper is the following. In sect. 2 we decsribe our Monte Carlo method for the photon bremsstrahlung calculation of a general semileptonic decay. This method is a substantial improvement compared to the method published in ref. 29. Sect. 3 contains the description of the photon bremsstrahlung amplitude of hyperon semileptonic decays, by using the Low-theorem. In sect. 4 the bremsstrahlung amplitude squared result is presented for unpolarized hyperon semileptonic decays. The model independent virtual correction and soft photon results are summarized in sect. 5. The results of our computer program runs are given in sect. 6. The high efficiency of our Monte Carlo method is illustrated, and the radiative correction results are compared to published analytical and numerical results of neutron, muon and hyperon semileptonic decays. The contributions of the various terms of the bremsstrahlung matrix element squared to the measurable unpolarized quantities are calculated, and the results are discussed. Finally, Appendix A and B contain useful formulae needed for our Monte Carlo algorithm.

\section{Monte Carlo method of photon bremsstrahlung calculation for semileptonic decays}

We start the presentation of our method with the calculation of the total decay rate of a semileptonic decay $P_{i} \rightarrow P_{f} l \bar{\nu}$. Here $l$ denotes a charged lepton, and $\nu$ is the neutrino counterpart of $l$. In the following the indices $1,2, i$ and $f$ refer to antineutrino, charged lepton, initial (decaying ) particle and final hadron ( or the other antineutrino in muon and $\tau$ decays ), respectively. $p, \underline{p, E}$ and $m$ denote four-momentum, three-momentum, energy and mass, respectively. The total decay rate ( up to order- $\alpha$ ) can be written as

$$
\rho=\rho_{0}+\rho_{V}(\lambda)+\rho_{B R}(\lambda)
$$

Here $\rho_{0}$ is the zeroth-order decay rate:

$$
\rho_{0}=\frac{1}{(2 \pi)^{5}} \frac{1}{2 m_{i}} \iiint d R_{3}\left(p_{i}, p_{f}, p_{2}, p_{1}\right) \frac{1}{2} \sum\left|\mathcal{M}_{0}\right|^{2}
$$

( $\sum$ denotes spin summation for all particles of the decay ),

$$
d R_{3}\left(p_{i}, p_{f}, p_{2}, p_{1}\right)=\frac{d^{3} \underline{p} f}{2 E_{f}} \frac{d^{3} \underline{p}-2}{2 E_{2}} \frac{d^{3} \underline{p}}{2 E_{1}} \delta^{4}\left(p_{i}-p_{f}-p_{2}-p_{1}\right)
$$


A similar formula holds for the $\rho_{V}(\lambda)$ virtual correction, but with $2 \sum \mathcal{R} e\left(\mathcal{M}_{0} \mathcal{M}_{\gamma}^{*}\right)$ instead of $\sum\left|\mathcal{M}_{0}\right|^{2}\left(\mathcal{M}_{\gamma}=\mathcal{M}_{\gamma}(\lambda)\right.$ is the virtual matrix element, $\lambda$ denotes the photon mass as infrared cutoff ). The bremsstrahlung part of the order- $\alpha$ electromagnetic correction can be expressed as

$$
\begin{gathered}
\rho_{B R}(\lambda)=\frac{1}{(2 \pi)^{8}} \frac{1}{2 m_{i}} \iiint \int d R_{4}\left(p_{i}, p_{f}, p_{2}, p_{1}, k\right) \frac{1}{2} \sum\left|\mathcal{M}_{B R}\right|^{2} \\
d R_{4}\left(p_{i}, p_{f}, p_{2}, p_{1}, k\right)=\frac{d^{3} \underline{p} f}{2 E_{f}} \frac{d^{3} \underline{p}-2}{2 E_{2}} \frac{d^{3} \underline{p}-1}{2 E_{1}} \frac{d^{3} \underline{k}}{2 k_{0}} \delta^{4}\left(p_{i}-p_{f}-p_{2}-p_{1}-k\right)
\end{gathered}
$$

Here $k$ denotes the bremsstrahlung photon four-momentum $\left(k_{0}=\sqrt{\underline{k}^{2}+\lambda^{2}}\right)$.

We introduce now the following function $(z \ll 1$ )

$$
\begin{gathered}
\omega\left(E_{2}\right):=\min \left\{z\left(E_{2}-m_{2}\right), z\left(m_{i}-E_{2}-E_{f m}\right)\right\} \\
E_{f m}=m_{f}+\frac{\left(m_{i}-m_{f}\right)^{2}-m_{2}^{2}}{2 m_{i}} .
\end{gathered}
$$

We split the bremsstrahlung phase space into soft and hard regions ( $K=|\underline{k}|)$ :

$$
\left.\begin{array}{ll}
K<\omega\left(E_{2}\right): & \text { soft photon events } \\
K>\omega\left(E_{2}\right): & \text { hard photon events }
\end{array}\right\} .
$$

Let us denote by $\mathcal{D}_{0}$ the zeroth-order Dalitz-plot of the $E_{2}$ and $E_{f}$ variables. The $\left(E_{2}, E_{f}\right)$ point is inside the $\mathcal{D}_{0}$ region if :

$$
\begin{gathered}
m_{2}<E_{2}<E_{2 m}, \\
E_{2 m}=\frac{m_{i}^{2}-m_{f}^{2}+m_{2}^{2}}{2 m_{i}}, \\
E_{f \min }\left(E_{2}\right)<E_{f}<E_{f \max }\left(E_{2}\right), \\
E_{f \min / \max }\left(E_{2}\right)=m_{f}+\frac{1}{2} \frac{\left(m_{i}-m_{f}-l_{ \pm}\right)^{2}}{m_{i}-l_{ \pm}}, \\
l_{ \pm}=E_{2} \pm\left|\underline{p}_{2}\right| .
\end{gathered}
$$

It is easy to show that for every $\left(E_{2}, E_{f}\right) \in \mathcal{D}_{0}$ point and for $z \ll 1$

$$
\omega\left(E_{2}\right) \ll \min \left\{E_{2}, E_{f}, Q_{0}\right\} \quad,
$$

where

$$
Q_{0}=m_{i}-E_{2}-E_{f} .
$$

Due to eq. (2.13) we can approximate the bremsstrahlung matrix element squared for soft photons as

$$
\sum\left|\mathcal{M}_{B R}\right|_{(\text {soft })}^{2} \approx-e^{2}\left(\frac{p_{2}}{\left(p_{2} \cdot k\right)}-\frac{p}{(p \cdot k)}\right)^{2} \sum\left|\mathcal{M}_{0}\right|^{2}
$$


where $p=p_{i}$ or $p=p_{f}$, depending on whether the $P_{i}$ or the $P_{f}$ particle is charged $\left(e^{2}=4 \pi \alpha\right)$.

The bremsstrahlung part of the total decay rate can be written as

$$
\rho_{B R}(\lambda)=\rho_{S}(z, \lambda)+\rho_{H}(z)
$$

where $\rho_{S}(z, \lambda)$ is the (2.4) integral for $K<\omega\left(E_{2}\right)$, and $\rho_{H}(z)$ is the same integral for $K>\omega\left(E_{2}\right)$ ( for hard photons we do not need the $\lambda$ infrared cutoff ). In order to calculate the $\rho_{S}(z, \lambda)$ soft part, we employ the $(2.15)$ approximation. $\rho_{S}(z, \lambda)$ can be written as a sum of a large and 2 small terms :

$$
\rho_{S}(z, \lambda)=\hat{\rho}_{S}(z, \lambda)+\rho_{I N}(z)+\rho_{O U T}(z)
$$

Here the first term contains the whole infrared divergence of the bremsstrahlung total decay rate, and it is also logarithmically divergent in the $z \rightarrow 0$ limit. For $z \ll 1$ it can be approximated as

$$
\begin{gathered}
\hat{\rho}_{S}(z, \lambda) \approx \int_{\mathcal{D}_{0}} \int d E_{2} d E_{f} Z_{S} W_{0}\left(E_{2}, E_{f}\right), \\
Z_{S}=-\frac{\alpha}{4 \pi^{2}} \int_{0}^{\hat{\omega}} \frac{d^{3} \underline{k}}{k_{0}}\left(\frac{p_{2}}{\left(p_{2} \cdot k\right)}-\frac{p}{(p \cdot k)}\right)^{2}, \\
\hat{\omega}=\hat{\omega}\left(E_{2}, E_{f}\right)=\min \left\{\omega\left(E_{2}\right),\left(Q_{0}-Q_{\min }\right) / 2\right\}, \\
Q_{\min }=|| \underline{p}_{2}|-| \underline{p}_{f}||, \\
W_{0}\left(E_{2}, E_{f}\right)=\frac{1}{128 m_{i} \pi^{3}} \sum\left|\mathcal{M}_{0}\right|^{2} .
\end{gathered}
$$

The result of the (2.19) integral is presented in appendix A.

The $\rho_{I N}(z)$ term can be expressed by a bremsstrahlung integral near the $E_{\text {fmin }}\left(E_{2}\right)$, $E_{2}>E_{2 h}$ curve, inside the $\mathcal{D}_{0}$ region, where

$$
E_{2 h}=\frac{1}{2}\left[m_{i}-m_{f}+\frac{m_{2}^{2}}{m_{i}-m_{f}}\right] \text {. }
$$

$\rho_{O U T}(z)$ denotes a similar integral near the $E_{f \min }\left(E_{2}\right), E_{2}<E_{2 h}$ curve, outside the $\mathcal{D}_{0}$ region. We have calculated these integrals, using the method described in app. B of ref. [18], and the contributions of these integrals to various distributions and to the total decay rate have been computed. These contributions decrease by decreasing the $z$ parameter, and for $z \sim 0.003$ they are negligible. Therefore, these integrals are not necessary for our method, and we do not present them in our paper.

The total decay rate can be approximately expressed as

$$
\rho \approx \rho_{0 V S}(z)+\rho_{H}(z)
$$


where

$$
\begin{gathered}
\rho_{0 V S}(z)=\int_{\mathcal{D}_{0}} \int d E_{2} d E_{f} W_{0 V S}\left(E_{2}, E_{f} ; z\right) \\
W_{0 V S}\left(E_{2}, E_{f} ; z\right)=W_{0}\left(E_{2}, E_{f}\right)+\frac{1}{64 m_{i} \pi^{3}} \sum \mathcal{R e}\left(\mathcal{M}_{0} \mathcal{M}_{\gamma}^{*}\right)+Z_{S} W_{0}\left(E_{2}, E_{f}\right) .
\end{gathered}
$$

The smaller is the $z$ parameter the better is the above approximation.

The calculation of the $\rho_{0 V S}(z)$ part is a trivial two-dimensional integration, if the $\mathcal{M}_{\gamma}$ virtual amplitude is known. For hyperon semileptonic decays $W_{0 V S}\left(E_{2}, E_{f} ; z\right)$ is a smooth function of $E_{2}$ and $E_{f}$, therefore the precise and quick numerical computation of the above integral is rather easy.

The situation is quite different in the case of $\rho_{H}(z)$. Here one has a 5 -dimensional integral, and the $\sum\left|\mathcal{M}_{B R}\right|^{2}$ integrand has large peaks (due to the charged particle propagators ):

a, infrared peak : for small $K$

$$
M_{B R}:=\sum\left|\mathcal{M}_{B R}\right|^{2} \sim \frac{1}{K^{2}}
$$

b, collinear peak : for $m_{2} \ll m_{i}-m_{f}, E_{2} \gg m_{2}, \theta \ll 1$

$$
M_{B R} \sim \frac{1}{\theta^{2}+m_{2}^{2} / E_{2}^{2}}
$$

( here $\theta$ is the angle between the photon and the electron directions in the decaying particle CMS ).

The precise computation of $\rho_{H}(z)$ by 5 -dimensional numerical integration needs a lot of computer time [28]. A semianalytical calculation method was desribed in ref. [16]. The computer time of the order- $\alpha$ total decay rate calculation by this method is rather short. On the other hand, by using this method, many complicated analytical integrals have to be calculated, and it is difficult to take into account the experimental details.

The Monte Carlo method is a very good tool for complicated many-dimensional integral calculation [30-39]. As it is well-known, huge isolated peaks of the integrand make the Monte Carlo method of integration very inefficient ( the presence of these peaks cause large statistical errors in the results ). One possibility to solve this problem is the employment of importance sampling. We have to find a $g$ function which approximates the $M_{B R}$ bremsstrahlung density rather well in the (2.27) and (2.28) peak regions, and we have to be able to generate hard bremsstrahlung events according to $g$ as a density function. The $g$ approximate function is good, if:

- the $w=M_{B R} / g$ weight function has small variance ;

- the event generation according to $g$ is simple and quick.

For this purpose, we consider the $P_{i} \rightarrow P_{f} l \bar{\nu} \gamma$ four-particle decay as two subsequent decays $[30-32]$ :

$$
P_{i} \rightarrow P_{f} l \bar{\nu} \gamma \quad: \quad P_{i} \rightarrow P_{M} l \gamma \quad, \quad P_{M} \rightarrow P_{f} \bar{\nu}
$$

The mass squared of the intermediate $P_{M}$ particle is

$$
M^{2}=\left(p_{f}+p_{1}\right)^{2}=\left(p_{i}-p_{2}-k\right)^{2} .
$$


The lower and upper limits of $M^{2}$ are

$$
M_{\text {min }}^{2}=m_{f}^{2} \quad, \quad M_{m a x}^{2}=\left(m_{i}-m_{2}\right)^{2} .
$$

The $\left(E_{2}, K\right)$ Dalitz region of the $P_{i} \rightarrow P_{M} l \gamma$ decay is the following :

$$
\begin{gathered}
m_{2}<E_{2}<E_{0} \\
E_{0}=\frac{m_{i}^{2}-M^{2}+m_{2}^{2}}{2 m_{i}}, \\
K_{\min }\left(E_{2}\right)<K<K_{\max }\left(E_{2}\right), \\
K_{\text {min } / \max }=K_{\min / \max }\left(E_{2}\right)=\frac{\Delta}{1-\frac{E_{2}}{m_{i}}(1 \mp \beta)}, \\
\Delta=E_{0}-E_{2} \quad, \quad \beta=\beta\left(E_{2}\right)=\frac{\left|\underline{p}_{2}\right|}{E_{2}}=\sqrt{1-m_{2}^{2} / E_{2}^{2}} .
\end{gathered}
$$

We are interested only in hard photon events, therefore we should generate $\left(E_{2}, K\right)$ events with $K>\omega\left(E_{2}\right)$. Let us denote by $E_{2}^{*}$ and $E_{2 \text { max }}$ those $E_{2}$ values where the $\omega\left(E_{2}\right)$ curve intersects the $K_{\min }\left(E_{2}\right)$ and $K_{\max }\left(E_{2}\right)$ boundaries, respectively. For $z \ll 1 \quad E_{2}^{*}$ and $E_{2 \max }$ are close to $E_{0}$, and we approximate the $\omega\left(E_{2}\right)$ function near $E_{0}$ by $\omega_{0}=\omega\left(E_{0}\right)$. The $E_{2}^{*}$ and $E_{2 \max }$ values can be calculated by iteration. For example :

$$
E_{2}^{*}=\frac{E_{0}-\omega_{0}}{1-\frac{\omega_{0}}{m_{i}}\left(1-\beta\left(E_{2}^{*}\right)\right)},
$$

and the starting value of the iteration could be $E_{2}^{*(0)}=E_{0}$.

Using the (2.29) composition of the four-particle decay into three- and two-particle decays, $\rho_{H}(z)$ can be written as $[32]$

$$
\begin{aligned}
& \rho_{H}(z)=\iint d \Omega_{1}^{*} \int_{M_{\min }^{2}}^{M_{\max }^{2}} d M^{2} \int_{m_{2}}^{E_{2 \max }} d E_{2} \int_{K_{m i n}^{\prime}}^{K_{\max }^{\prime}} d K F_{B R} \\
& F_{B R}=\frac{1}{2^{13} \pi^{6} m_{i}} a\left(M^{2}\right) M_{B R}, \\
& a\left(M^{2}\right)=\frac{M^{2}-m_{f}^{2}}{M^{2}}, \\
& K_{\min }^{\prime}=\left\{\begin{array}{lll}
K_{\min } & : \text { if } & E_{2}<E_{2}^{*} \\
\omega_{0}=\omega\left(E_{0}\right) & : \text { if } & E_{2}>E_{2}^{*}
\end{array} .\right.
\end{aligned}
$$

The $\Omega_{1}^{*}$ solid angle describes the orientation of the antineutrino three-momentum in the CMS of the $P_{M}$ particle.

We define now the above mentioned approximate function as

$$
g:=\frac{a\left(M^{2}\right)}{K\left(p_{2} \cdot k\right)}=\frac{a\left(M^{2}\right)}{E_{2} K^{2}(1-\beta \cos \theta)} .
$$


It can easily be shown that

$$
g=\frac{a\left(M^{2}\right)}{m_{i} K(K-\Delta)} .
$$

Let us introduce the following functions:

$$
\begin{gathered}
f\left(E_{2}\right)=\int_{K_{\min }^{\prime}}^{K_{\max }} \frac{d K}{K(K-\Delta)}, \\
F\left(M^{2}\right)=a\left(M^{2}\right) \int_{m_{2}}^{E_{2 \max }} d E_{2} f\left(E_{2}\right) .
\end{gathered}
$$

For $E_{2}<E_{2}^{*}$ :

$$
f\left(E_{2}\right)=\frac{1}{\Delta} \ln \left(\frac{1+\beta}{1-\beta}\right),
$$

and for $E_{2}^{*}<E_{2}<E_{2 \max }$ :

$$
f\left(E_{2}\right)=\frac{1}{\Delta}\left\{\ln \left(\frac{E_{2}}{m_{i}}\right)+\ln (1+\beta)-\ln \left(\frac{\omega_{0}-\Delta}{\omega_{0}}\right)\right\} .
$$

An efficient numerical calculation of $F\left(M^{2}\right)$ is the following :

$$
\begin{gathered}
F\left(M^{2}\right)=a\left(M^{2}\right)\left(F_{1}+F_{2}\right), \\
F_{1}=t^{*} \ln \left(\frac{E_{0}-m_{2}}{E_{0}-E_{2}^{*}}\right)+\int_{m_{2}}^{E_{2}^{*}} d E_{2} \frac{t\left(E_{2}\right)-t^{*}}{E_{0}-E_{2}}, \\
F_{2}=\int_{E_{2}^{*}}^{E_{2 \max }} d E_{2} f\left(E_{2}\right), \\
t\left(E_{2}\right)=\ln \left(\frac{1+\beta}{1-\beta}\right), t^{*}=t\left(E_{2}^{*}\right) .
\end{gathered}
$$

The generation algorithm of the hard bremsstrahlung events according to the $g$ function is the following :

1. We generate an $M^{2}$ value according to $F\left(M^{2}\right)$ as ( unnormalized ) probability density function, using the rejection method of von Neumann. The $F\left(M^{2}\right)$ function is computed in 500 points before the beginning of the generation, the maximum value of $F\left(M^{2}\right)$ is estimated, and during the generation we use linear interpolation for the $F\left(M^{2}\right)$ calculation.

2. For the $E_{2}$ generation according to $f\left(E_{2}\right)$ we first introduce the

$$
h\left(E_{2}\right)=\frac{1}{E_{0}-E_{2}}
$$

function, and we generate $E_{2}$ points according to the $h\left(E_{2}\right)$ distribution between $m_{2}$ and $E_{2 \max }$ :

$$
E_{2}=E_{0}-\left(E_{0}-m_{2}\right) e^{-u_{1} H},
$$




$$
H=\int_{m_{2}}^{E_{2 \max }} \frac{d E_{2}}{E_{0}-E_{2}}=\ln \left(\frac{E_{0}-m_{2}}{E_{0}-E_{2 \max }}\right) .
$$

Here and below $u_{j}(j=1,2, \ldots)$ denote uniform, independent random numbers in the $[0,1]$ interval. Then the Neumann rejection method is employed with the $w^{\prime}=\left(E_{0}-E_{2}\right) f\left(E_{2}\right)$ weight function.

3. We generate the $K$ valus as

$$
\begin{gathered}
K=\frac{\Delta}{1-e^{C}} \\
C=u_{2} f\left(E_{2}\right) \Delta+\ln \left(\frac{K_{\text {min }}^{\prime}-\Delta}{K_{\text {min }}^{\prime}}\right) .
\end{gathered}
$$

4. The generation of the $\cos \theta_{1}^{*}$ and $\phi_{1}^{*}$ values of the $\Omega_{1}^{*}$ solid angle is trivial :

$$
\begin{aligned}
\cos \theta_{1}^{*} & =2 u_{3}-1, \\
\phi_{1}^{*} & =2 \pi u_{4} .
\end{aligned}
$$

The $M^{2}, E_{2}, K, \cos \theta_{1}^{*}$ and $\phi_{1}^{*}$ values determine a hard bremsstrahlung event. The Monte Carlo weight of this event is defined as

$$
w:=\frac{F_{B R}}{g}=\frac{K\left(p_{2} \cdot k\right)}{2^{13} \pi^{6} m_{i}} M_{B R} .
$$

The above event generation procedure is repeated $N_{H}$ times, and the weight for each event is calculated ( $w_{j}$ for the $j$-th event ). The Monte Carlo estimate of the (2.38) integral is

$$
\rho_{H}(z) \approx \frac{V_{g}}{N_{H}} \sum_{j=1}^{N_{H}} w_{j}
$$

and the estimate for the statistical error of (2.59) is

$$
\delta \rho_{H}(z) \approx \frac{V_{g}}{N_{H}} \sqrt{\sum_{j=1}^{N_{H}} w_{j}^{2}-\frac{1}{N_{H}}\left(\sum_{j=1}^{N_{H}} w_{j}\right)^{2}}
$$

where

$$
V_{g}=\int_{-1}^{1} \int_{0}^{2 \pi} d \Omega_{1}^{*} \int_{M_{m i n}^{2}}^{M_{\max }^{2}} d M^{2} \int_{m_{2}}^{E_{2 \max }} d E_{2} \int_{K_{\min }^{\prime}}^{K_{\max }} d K g=\frac{4 \pi}{m_{i}} \int_{M_{\min }^{2}}^{M_{\max }^{2}} d M^{2} F\left(M^{2}\right)
$$

The $M_{B R}$ bremsstrahlung matrix element squared is usually expressed as a function of the scalar products of the $p_{i}, p_{f}, p_{2}, p_{1}$ and $k$ four-momenta. We present these scalar products as functions of the $M^{2}, E_{2}, K, \cos \theta_{1}^{*}$ and $\phi_{1}^{*}$ Monte Carlo variables in appendix B.

For the generation of unweighted events the following procedure can be employed [35,37,39]. First, we compute the $\rho_{0 V S}(z)$ "soft decay rate" by two-dimensional numerical integration 
( see eq. (2.25)), and the $\rho_{H}(z)$ "hard decay rate" by the above described Monte Carlo method ( $z<<1$; f.e.: $z=10^{-3}$ ). We define the $P_{H}$ number as

$$
P_{H}:=\frac{\rho_{H}(z)}{\rho_{0 V S}(z)+\rho_{H}(z)} .
$$

In order to generate an unweighted decay event, we compare $P_{H}$ with a $u \in[0,1]$ random number. If $P_{H}<u$, then we generate a soft decay event, with zeroth-order kinematics. This can be achieved by $\left(E_{2}, E_{f}\right)$ generation on $\mathcal{D}_{0}$, using the Neumann rejection method with the $W_{0 V S}\left(E_{2}, E_{f} ; z\right)$ function. If $P_{H}>u$, then we generate a hard decay event, i.e. a decay with hard bremsstrahlung photon. For this purpose, we generate hard bremsstrahlung events according to the $g$ density function, and then we use the Neumann method with the $w=F_{B R} / g$ function. The $w_{\max }$ maximum value of the $w$ weight function can be estimated during the $\rho_{H}(z)$ calculation.

Let us define the efficiencies of the soft and the hard Monte Carlo generations as [39]

$$
\begin{aligned}
E_{0 V S} & :=100 \frac{<W_{0 V S}>}{W_{0 V S}^{\text {max }}}, \\
E_{H} & :=100 \frac{<w>}{w_{\text {max }}},
\end{aligned}
$$

where $\left\langle W_{0 V S}\right\rangle$ and $\left\langle w>\right.$ are the Monte Carlo average values of the $W_{0 V S}\left(E_{2}, E_{f} ; z\right)$ and the $w$ functions :

$$
<W_{0 V S}>=\frac{1}{N_{S}} \sum_{j=1}^{N_{S}} W_{0 V S j} \quad, \quad<w>=\frac{1}{N_{H}} \sum_{j=1}^{N_{H}} w_{j}
$$

( $N_{S}$ and $N_{H}$ are the numbers of soft and hard Monte Carlo generated events ), and $W_{0 V S}^{\max }$, $w_{\text {max }}$ are the maximum values of these functions. The efficiency measures ( in $\%$ ) the proportion of the accepted events of the rejection method ( compared to the total number of events ). We will see in sect. 6 that both the $E_{0 V S}$ and the $E_{H}$ efficiencies are large ( in the case of hyperon semileptonic decays ). Therefore, our method is useful for unweighted event generation in experimental off-line analyses.

On the other hand, one can use our method also for generation of weighted events. In the experimental analyses it is important to calculate precise theoretical bin distributions, which are compared to the measured bin distributions. These bin distributions are usually normalized, because only the shape of these distributions is relevant for the fit analysis ( see refs. [1-4]). Let us define the one-dimensional normalized bin distribution of the $x$ kinematic parameter by

$$
\begin{gathered}
b(i)=n \frac{\int_{x_{i}}^{x_{i+1}} d x W(x)}{\rho} \quad(i=1, \ldots, n), \\
\rho=\int_{x_{1}}^{x_{n+1}} d x W(x)=\rho_{0 V S}(z)+\rho_{H}(z),
\end{gathered}
$$


where $W(x)$ is the distribution of $x$ ( with radiative correction included ), $\rho$ is the total decay rate, $n$ is the number of bins, and $x_{i}(i=1, \ldots, n+1)$ define the bin intervals $\left(x_{1}\right.$ and $x_{n+1}$ are the minimal and maximal values of $x$ ). The $b(i)$ bin distribution can be calculated by the following equation :

$$
\begin{gathered}
b(i)=\frac{100-r_{H}(z)}{100} b_{0 V S}(z ; i)+\frac{r_{H}(z)}{100} b_{H}(z ; i), \\
r_{H}(z)=100 \frac{\rho_{H}(z)}{\rho_{0 V S}(z)+\rho_{H}(z)} .
\end{gathered}
$$

Here $b_{0 V S}(z ; i)$ and $b_{H}(z ; i)$ are the corresponding bin distributions of the soft and the hard decay events, respectively. One can compute them by weighted event generation. Let us see, for example, the calculation of $b_{H}(z ; i)$. We generate $N_{H}$ hard events according to $g$. Let us denote the $x$ value of the $j^{\prime}$ th event by $x(j)$, and the $w=F_{B R} / g$ weight of this event by $w_{j}$. Then

$$
b_{H}(z ; i)=n \frac{\sum_{j=1}^{N_{H}} \theta_{i}(x(j)) w_{j}}{\sum_{j=1}^{N_{H}} w_{j}},
$$

where

$$
\theta_{i}(x)=\left\{\begin{array}{lll}
1 & : \text { if } & x_{i} \leq x<x_{i+1} \\
0 & : \text { if } & x<x_{i} \text { or } x \geq x_{i+1}
\end{array} .\right.
$$

The $b_{0 V S}(z ; i)$ can be similarly calculated ( by soft event generation ). For special $x$ parameters ( f.e.: electron energy ) it can also be computed by numerical integration ( this requires less computer time, if one would like to achieve high precision ).

\section{The photon bremsstrahlung amplitude}

Electromagnetic current conservation enables one to calculate the photon bremsstrahlung amplitude in hadronic processes not only to order $K^{-1}$, but also to order $K^{0}$ [22-27]. We write the photon bremsstrahlung amplitude for the $B_{i} \rightarrow B_{f} e \bar{\nu}_{e}$ hyperon semileptonic decay ( $B_{i}$ and $B_{f}$ denote quasi-stable SU(3)-octet baryons) as a sum of three amplitude terms:

$$
\mathcal{M}_{B R}=\tilde{\mathcal{M}}+\mathcal{M}_{M M}+\delta \mathcal{M}
$$

Here $\tilde{\mathcal{M}}$ is the dominant part of the bremsstrahlung amplitude :

$$
\tilde{\mathcal{M}}=e(P \varepsilon) \mathcal{M}_{0}(q)+\mathcal{M}_{e}\left[K^{0}\right]
$$

where $e$ is the electric charge $\left(e>0, e^{2}=4 \pi \alpha\right), \varepsilon$ is the bremsstrahlung photon polarization vector. The $P$ four-vector is defined as

$$
P=\frac{p}{(p \cdot k)}-\frac{p_{2}}{\left(p_{2} \cdot k\right)}
$$

where $p_{2}$ and $k$ are the electron and photon four-momenta, respectively. $p$ is the fourmomentum of the charged baryon: $p=p_{i}$ for decays of charged hyperons ( like $\Sigma^{-} \rightarrow n e \bar{\nu}$ ), 
and $p=p_{f}$ for neutral hyperon decays ( like $\Lambda \rightarrow p e \bar{\nu}$ ), where $p_{i}$ and $p_{f}$ are the four-momenta of the initial ( decaying ) and final baryons, respectively.

$\mathcal{M}_{0}(q)$ is the zeroth-order decay amplitude:

$$
\begin{gathered}
\mathcal{M}_{0}(q)=\frac{G}{\sqrt{2}} h_{\rho}(q) l^{\rho}, \\
l^{\rho}=\bar{u}_{2} \gamma^{\rho}\left(1-\gamma_{5}\right) v_{1}, \\
h_{\rho}(q)=\bar{u}_{f} H_{\rho}(q) u_{i}, \\
H_{\rho}(q)=f_{1}\left(1+\lambda_{f} q^{2}\right) \gamma_{\rho}+g_{1}\left(1+\lambda_{g} q^{2}\right) \gamma_{\rho} \gamma_{5}+\frac{f_{2}}{2 m_{i}}\left[\gamma_{\rho}, \phi\right], \\
q=p_{i}-p_{f} .
\end{gathered}
$$

In these expressions the indices $1,2, i$ and $f$ refer to antineutrino, electron, initial baryon and final baryon, respectively. $G=G_{\mu} V_{u d}$ for strangeness-conserving decays, $G=G_{\mu} V_{u s}$ for strangeness-changing decays, where $G_{\mu}$ is the muon decay coupling constant, $V_{u d}$ and $V_{u s}$ are Cabibbo-Kobayashi-Maskawa matrix elements. $\lambda_{f}=2 / M_{V}^{2}$ and $\lambda_{g}=2 / M_{A}^{2}$, where $M_{V}=840 \mathrm{MeV}, M_{A}=1080 \mathrm{MeV}$ for strangeness-conserving decays, and $M_{V}=980 \mathrm{MeV}$, $M_{A}=1250 \mathrm{MeV}$ for strangeness-changing decays [40]. In (3.7) we have omitted the $f_{3}, g_{2}$ and $g_{3}$ form factors ( see section 4 for comments ). Time reversal invariance is assumed, therefore the form factors are supposed to be real. The $q^{2}$ dependence of the $f_{1}\left(q^{2}\right)$ and $g_{1}\left(q^{2}\right)$ form factors has large $(\sim 10 \%)$ effect on the measurable zeroth-order distributions, therefore we have included it in $(3.7)$.

The $e(P \varepsilon) \mathcal{M}_{0}(q)$ term has $1 / K$ photon energy dependence, and is therefore infrared divergent. All the other terms in $\mathcal{M}_{B R}$ are finite in the $K \rightarrow 0$ limit.

The second term in (3.2) is the order- $K^{0}$ part of the electron-bremsstrahlung amplitude:

$$
\mathcal{M}_{e}\left[K^{0}\right]=-\frac{G}{\sqrt{2}} e \varepsilon_{\mu} h_{\rho}(q) \frac{\bar{u}_{2} \gamma^{\mu} k \gamma^{\rho}\left(1-\gamma_{5}\right) v_{1}}{2\left(p_{2} \cdot k\right)}
$$

The $\mathcal{M}_{M M}$ and $\delta \mathcal{M}$ terms in (3.1) are of order $\Delta m / m_{i}$ compared to $\tilde{\mathcal{M}}$ $\left(\Delta m=m_{i}-m_{f}\right)$. The $\mathcal{M}_{M M}$ magnetic moment term can be written as the sum of Dirac and anomalous magnetic moment contributions:

$$
\mathcal{M}_{M M}=\mathcal{M}_{D}+\mathcal{M}_{A M}
$$

Here $\mathcal{M}_{D}$ is similar to $(3.9)$ :

$$
\mathcal{M}_{D}=\frac{G}{\sqrt{2}} e \varepsilon^{\mu} l^{\rho} \frac{\bar{u}_{f} \Gamma_{D} u_{i}}{2(p \cdot k)}
$$

where for charged hyperon decays

$$
\Gamma_{D}=H_{\rho}(0) \gamma_{\mu} k k,
$$

and for neutral hyperon decays

$$
\Gamma_{D}=\gamma_{\mu} k H_{\rho}(0)
$$


The $\mathcal{M}_{D}$ part of the bremsstrahlung amplitude is of order $\Delta m / m_{i}$ compared to the dominant $\tilde{\mathcal{M}}$, therefore we have neglected the $q$-dependent terms of $H_{\rho}$ in $\Gamma_{D}$. These terms would yield contributions to $\mathcal{M}_{B R}$ of the order of $\left(\Delta m / m_{i}\right)^{2}$, but this is the order of magnitude of the terms of order- $K$ in $\mathcal{M}_{B R}$, which cannot be calculated rigorously by the Low-theorem (the order- $K$ terms in $\mathcal{M}_{B R}$ are model dependent ).

The $\tilde{\mathcal{M}}+\mathcal{M}_{D}$ part defines the pointlike hadron approximation for the photon bremsstrahlung amplitude ( the initial and final baryons are treated as pointlike Dirac particles, just like the electron ).

The interaction of the bremsstrahlung photon with the anomalous magnetic moments of the baryons is taken into account by the $\mathcal{M}_{A M}$ term:

$$
\begin{gathered}
\mathcal{M}_{A M}=\mathcal{M}_{A M}^{i}+\mathcal{M}_{A M}^{f}, \\
\mathcal{M}_{A M}^{i}=-\frac{G}{\sqrt{2}} e \varepsilon^{\mu} l^{\rho} \frac{\hat{\kappa}_{i}}{2 m_{i}} \frac{\bar{u}_{f} H_{\rho}(0)\left(p_{i}+m_{i}\right) \gamma_{\mu} \mid k u_{i}}{2\left(p_{i} \cdot k\right)}, \\
\mathcal{M}_{A M}^{f}=\frac{G}{\sqrt{2}} e \varepsilon^{\mu} l^{\rho} \frac{\hat{\kappa}_{f}}{2 m_{f}} \frac{\bar{u}_{f} \gamma_{\mu} \not k\left(\phi_{f}+m_{f}\right) H_{\rho}(0) u_{i}}{2\left(p_{f} \cdot k\right)}
\end{gathered}
$$

Here $\hat{\kappa}_{i}$ and $\hat{\kappa}_{f}$ are the anomalous magnetic moments of the baryons in $e /\left(2 m_{i}\right)$ and $e /\left(2 m_{f}\right)$ units, respectively. For example:

$$
\begin{gathered}
\hat{\kappa}_{p}=1.79 \quad, \quad \hat{\kappa}_{n}=-1.91, \\
\hat{\kappa}_{\Sigma^{-}}=-0.47 \quad, \quad \hat{\kappa}_{\Lambda}=-0.72 .
\end{gathered}
$$

The above expressions can be rewritten as

$$
\begin{gathered}
\mathcal{M}_{A M}^{i}=-\hat{\kappa}_{i} \mathcal{M}_{D}^{i}+\frac{G}{\sqrt{2}} e \varepsilon^{\mu} l^{\rho} \frac{\hat{\kappa}_{i}}{2 m_{i}} \bar{u}_{f} H_{\rho}(0) G_{\mu} u_{i} \\
\mathcal{M}_{A M}^{f}=\hat{\kappa}_{f} \mathcal{M}_{D}^{f}+\frac{G}{\sqrt{2}} e \varepsilon^{\mu} l^{\rho} \frac{\hat{\kappa}_{f}}{2 m_{f}} \bar{u}_{f} G_{\mu} H_{\rho}(0) u_{i} \\
G_{\mu}=\gamma_{\mu}-\frac{p}{(p \cdot k)} \not k
\end{gathered}
$$

Here $\mathcal{M}_{D}^{i}$ is expression (3.11) with (3.12), and $\mathcal{M}_{D}^{f}$ denotes expression (3.11) with (3.13).

Finally, $\delta \mathcal{M}$ contains the derivative terms :

$$
\begin{gathered}
\delta \mathcal{M}=\delta \mathcal{M}\left[q^{2}\right]+\delta \mathcal{M}\left[f_{2}\right] \\
\delta \mathcal{M}\left[q^{2}\right]=2 \frac{G}{\sqrt{2}} e(Q \varepsilon) l^{\rho} \bar{u}_{f}\left[f_{1} \lambda_{f} \gamma_{\rho}+g_{1} \lambda_{g} \gamma_{\rho} \gamma_{5}\right] u_{i} \\
Q=q-\frac{(q \cdot k)}{(p \cdot k)} p \\
\delta \mathcal{M}\left[f_{2}\right]=\frac{G}{\sqrt{2}} e \varepsilon^{\mu} l^{\rho} \frac{f_{2}}{2 m_{i}} \bar{u}_{f}\left[\gamma_{\rho}, G_{\mu}\right] u_{i}
\end{gathered}
$$


The photon bremsstrahlung amplitude of hyperon semileptonic decays derived by the Low-theorem can be found in refs. $[13,17,19,20]$. In these papers the $f_{3}, g_{2}$ and $g_{3}$ form factor terms were included in the amplitude expressions, but the $q^{2}$ dependence of the $f_{1}\left(q^{2}\right)$ and $g_{1}\left(q^{2}\right)$ form factors was neglected. Our $\tilde{\mathcal{M}}, \mathcal{M}_{M M}$ and $\delta \mathcal{M}\left[f_{2}\right]$ terms agree with the corresponding amplitude terms of the above mentioned papers ( as far as $e$ is defined to be negative in these papers ).

\section{Bremsstrahlung matrix element squared for unpolarized decays}

The matrix element squared in the case of unpolarized hyperon semileptonic decays has been calculated by means of the symbolic algebraic program REDUCE. The terms of order $\left(\Delta m / m_{i}\right)^{2}$ have been neglected. We write :

$$
\sum\left|\mathcal{M}_{B R}\right|^{2}=M_{B R}=G^{2} e^{2} \sum_{j=1}^{13} C_{j}
$$

The first term in this sum comes from the square of the $e(P \varepsilon) \mathcal{M}_{0}(q)$ amplitude term:

$$
C_{1}=-32 P^{2} L_{0}
$$

For charged hyperon decays

$$
P^{2}=\frac{m_{i}^{2}}{C^{2}}+\frac{m_{2}^{2}}{F^{2}}-2 \frac{B}{C F},
$$

and for neutral hyperon decays

$$
P^{2}=\frac{m_{f}^{2}}{W^{2}}+\frac{m_{2}^{2}}{F^{2}}-2 \frac{Z}{W F} .
$$

Here and below $A, B, C, D, E, F, X, Y, Z$ and $W$ denote the 10 scalar products of the $p_{i}, p_{1}, p_{2}, k$ and $p_{f}$ four-momenta ( see appendix B ).

The definition of $L_{0}$ is

$$
L_{0}:=\frac{1}{32 G^{2}} \sum\left|\mathcal{M}_{0}(q)\right|^{2}
$$

It is a quadratic function of the form factors ( see also section II and app. A in ref. [18]):

$$
\begin{aligned}
L_{0} & =f_{1}^{2}\left(q^{2}\right) L_{0}\left[f_{1}^{2}\right]+g_{1}^{2}\left(q^{2}\right) L_{0}\left[g_{1}^{2}\right]+f_{1}\left(q^{2}\right) g_{1}\left(q^{2}\right) L_{0}\left[f_{1} g_{1}\right]+ \\
& +f_{2} g_{1}\left(q^{2}\right) L_{0}\left[f_{2} g_{1}\right]+f_{1}\left(q^{2}\right) f_{2} L_{0}\left[f_{1} f_{2}\right]+f_{2}^{2} L_{0}\left[f_{2}^{2}\right]
\end{aligned}
$$

where

$$
\begin{gathered}
f_{1}\left(q^{2}\right)=f_{1}\left(1+\lambda_{f} q^{2}\right) \quad, \quad g_{1}\left(q^{2}\right)=g_{1}\left(1+\lambda_{g} q^{2}\right), \\
q^{2}=\left(p_{i}-p_{f}\right)^{2}=m_{i}^{2}+m_{f}^{2}-2 X .
\end{gathered}
$$

The coefficients of the various form factor combinations are the following:

$$
L_{0}\left[f_{1}^{2}\right]=B Y+A Z-m_{i} m_{f} D
$$




$$
\begin{gathered}
L_{0}\left[g_{1}^{2}\right]=B Y+A Z+m_{i} m_{f} D \\
L_{0}\left[f_{1} g_{1}\right]=2(B Y-A Z) \\
L_{0}\left[f_{2} g_{1}\right]=\frac{m_{i}+m_{f}}{m_{i}} L_{0}\left[f_{1} g_{1}\right] \\
L_{0}\left[f_{1} f_{2}\right]=\frac{1}{m_{i}}\left\{m_{i}\left[Q_{2} Y+Q_{1} Z+Q_{f} D\right]-m_{f}\left[Q_{2} A+Q_{1} B+Q_{i} D\right]\right\} \\
L_{0}\left[f_{2}^{2}\right]=\frac{1}{m_{i}^{2}}\left\{Q_{i} Q_{2} Y+Q_{i} Q_{1} Z+Q_{2} Q_{f} A+Q_{1} Q_{f} B-Q_{1} Q_{2} X-\right. \\
\left.-m_{i} m_{f} Q_{1} Q_{2}+\frac{1}{2} q^{2}\left[D X-2 B Y-2 A Z-m_{i} m_{f} D\right]\right\} \\
Q_{1}=\left(p_{1} \cdot q\right)=A-Y \quad, \quad Q_{2}=\left(p_{2} \cdot q\right)=B-Z \\
Q_{i}=\left(p_{i} \cdot q\right)=m_{i}^{2}-X \quad, \quad Q_{f}=\left(p_{f} \cdot q\right)=X-m_{f}^{2}
\end{gathered}
$$

$C_{1}$ is the only infrared divergent term among the 13 terms in the sum of (4.1). It is proportional to the zeroth-order matrix element squared, and this fact ensures the cancellation of the infrared divergences in the sum of the virtual and bremsstrahlung corrections. We have not discarded the $L_{0}\left[f_{1} f_{2}\right]$ and $L_{0}\left[f_{2}^{2}\right]$ terms, which are of order $\left(\Delta m / m_{i}\right)^{2}$, in order to maintain the precise proportionality of $C_{1}$ to $\sum\left|\mathcal{M}_{0}(q)\right|^{2}$. If someone includes the $f_{3}$, $g_{2}$ and $g_{3}$ form factors into the zeroth-order expressions, then the additional form factor combinations, containing the $f_{3}, g_{2}$ and $g_{3}$ form factors, should also be added to eq. (4.6).

The $C_{2}$ term represents the interference of the two amplitude terms in (3.2):

$$
\begin{gathered}
C_{2}=\frac{1}{F}\left\{f_{1}^{2}\left(q^{2}\right) S_{1}\left(m_{f}\right)+g_{1}^{2}\left(q^{2}\right) S_{1}\left(-m_{f}\right)+f_{1} g_{1} S_{2}+f_{2} g_{1} S_{3}\right\}, \\
S_{1}\left(m_{f}\right)=-32\left\{A F R_{f}-A W R_{2}-C Y R_{2}+F Y R_{i}+m_{i} m_{f}\left[E R_{2}-F R_{1}\right]\right\}, \\
S_{2}=64\left[A F R_{f}-A W R_{2}+C Y R_{2}-F Y R_{i}\right], \\
S_{3}=2 S_{2},
\end{gathered}
$$

where

$$
R_{j}=\left(p_{j} \cdot P\right) \quad(j=i, f, 1,2) .
$$

For charged hyperon decays :

$$
\begin{array}{ll}
R_{i}=\frac{m_{i}^{2}}{C}-\frac{B}{F} \quad, \quad R_{f}=\frac{X}{C}-\frac{Z}{F}, \\
R_{1}=\frac{A}{C}-\frac{D}{F} \quad, \quad R_{2}=\frac{B}{C}-\frac{m_{2}^{2}}{F} .
\end{array}
$$

For neutral hyperon decays :

$$
\begin{array}{ll}
R_{i}=\frac{X}{W}-\frac{B}{F} \quad, & R_{f}=\frac{m_{f}^{2}}{W}-\frac{Z}{F}, \\
R_{1}=\frac{Y}{W}-\frac{D}{F} \quad, & R_{2}=\frac{Z}{W}-\frac{m_{2}^{2}}{F} .
\end{array}
$$


The $C_{3}$ term is proportional to the square of the $\mathcal{M}_{e}\left[K^{0}\right]$ amplitude:

$$
C_{3}=\frac{32}{F} L_{0}\left[p_{2} \rightarrow k\right]
$$

One can obtain $L_{0}\left[p_{2} \rightarrow k\right]$ by substituting the $p_{2}$ four-momenta by $k$ in the expressions of app. A in ref. [18]. A good approximation is

$$
\begin{aligned}
L_{0}\left[p_{2} \rightarrow k\right]=f_{1}^{2}\left(q^{2}\right)(C Y & \left.+A W-m_{i} m_{f} E\right)+g_{1}^{2}\left(q^{2}\right)\left(C Y+A W+m_{i} m_{f} E\right)+ \\
& +2\left(f_{1}+2 f_{2}\right) g_{1}(C Y-A W) .
\end{aligned}
$$

The $C_{4}$ and $C_{5}$ terms represent the interference of $\mathcal{M}_{D}$ with the first and second parts of (3.2), respectively. For charged hyperon decays :

$$
\begin{gathered}
C_{4}=\frac{1}{C}\left[f_{1}^{2} S_{4}+g_{1}^{2} S_{4}+f_{1} g_{1} S_{5}\right], \\
C_{5}=\frac{1}{2 F C}\left[f_{1}^{2} S_{6}\left(m_{f}\right)+g_{1}^{2} S_{6}\left(-m_{f}\right)+f_{1} g_{1} S_{7}\right],
\end{gathered}
$$

where

$$
\begin{gathered}
S_{4}=-32\left[A C R_{2}-A F R_{i}+B C R_{1}-B E R_{i}\right], \\
S_{5}=-64\left[A C R_{2}-A F R_{i}-B C R_{1}+B E R_{i}\right], \\
S_{6}\left(m_{f}\right)=-64 F\left(2 C Y-m_{i} m_{f} E\right), \\
S_{7}=-256 F A C .
\end{gathered}
$$

For neutral hyperon decays:

$$
\begin{gathered}
C_{4}=-\frac{1}{W}\left[f_{1}^{2} S_{4}+g_{1}^{2} S_{4}-f_{1} g_{1} S_{5}\right], \\
C_{5}=-\frac{1}{2 F W}\left[f_{1}^{2} S_{6}\left(m_{f}\right)+g_{1}^{2} S_{6}\left(-m_{f}\right)-f_{1} g_{1} S_{7}\right] .
\end{gathered}
$$

The $C_{6}-C_{9}$ terms are contributions from the anomalous magnetic moments of the baryons. The interference of $\mathcal{M}_{A M}$ with $e(P \varepsilon) \mathcal{M}_{0}$ is of order $\left(\Delta m / m_{i}\right)^{2}$, therefore we neglect it. This is in accordance with the Burnett-Kroll theorem [41,42]. On the other hand, the interference of $\mathcal{M}_{A M}$ with $\mathcal{M}_{e}\left[K^{0}\right]$ is of order $\Delta m / m_{i}$, and this cannot be neglected [13]. The $C_{6}$ and $C_{7}$ terms can be expressed by the help of the $C_{5}$ term. For charged hyperon decays:

$$
C_{6}=-\hat{\kappa}_{i} C_{5} \quad, \quad C_{7}=-\hat{\kappa}_{f} C_{5}\left[g_{1} \rightarrow-g_{1}\right]
$$

( see eq. (4.26)), and for neutral hyperon decays :

$$
C_{6}=\hat{\kappa}_{f} C_{5} \quad, \quad C_{7}=\hat{\kappa}_{i} C_{5}\left[g_{1} \rightarrow-g_{1}\right]
$$

( see eq. (4.32)). 
The expressions for $C_{8}$ and $C_{9}$ are the following:

$$
\begin{gathered}
C_{8}=\frac{\hat{\kappa}_{i}}{2 m_{i} F}\left[f_{1}^{2} S_{8}\left(m_{f}\right)+g_{1}^{2} S_{8}\left(-m_{f}\right)+f_{1} g_{1} S_{9}\right], \\
C_{9}=\frac{\hat{\kappa}_{f}}{2 m_{f} F}\left[f_{1}^{2} S_{8}\left(m_{f}\right)+g_{1}^{2} S_{8}\left(-m_{f}\right)-f_{1} g_{1} S_{9}\right], \\
S_{8}\left(m_{f}\right)=-32\left\{m_{i}\left[3 A F-C D+B E-m_{i}^{2} \frac{E F}{C}\right]+\right. \\
\left.+m_{f}\left[A F-B E+C D-m_{i}^{2} \frac{E F}{C}\right]\right\}, \\
S_{9}=-64 m_{i}\left[A F-B E+C D+m_{i}^{2} \frac{E F}{C}\right] .
\end{gathered}
$$

The terms $C_{10}-C_{13}$ contain the interference of the $\delta \mathcal{M}$ derivative amplitude with $\tilde{\mathcal{M}}$ :

$$
\begin{gathered}
C_{10}=-128(P Q)\left\{f_{1}^{2} \lambda_{f} L_{0}\left[f_{1}^{2}\right]+g_{1}^{2} \lambda_{g} L_{0}\left[g_{1}^{2}\right]\right\} \\
Q=q-\frac{(q \cdot k)}{\left(p_{i} \cdot k\right)} p_{i}, \\
(P Q)=R_{i}-R_{f}-\frac{Q_{k}}{C} R_{i}, \\
Q_{k}=(q \cdot k)=C-W \quad, \\
C_{11}=\frac{2}{F}\left[f_{1}^{2} \lambda_{f}\left(S_{10}+S_{11}\right)+g_{1}^{2} \lambda_{g}\left(S_{10}-S_{11}\right)\right], \\
S_{10}=64 A\left(C T_{2}-F T_{i}\right), \\
S_{11}=32 m_{i}^{2}\left(F T_{1}-E T_{2}\right), \\
T_{1}=Q_{1}-\frac{Q_{k}}{C} A \quad, \\
T_{2}=Q_{2}-\frac{Q_{k}}{C} B \quad, \\
T_{i}=Q_{i}-\frac{Q_{k}}{C} m_{i}^{2} \quad, \\
C_{12}=-128 f_{2} g_{1}\left[A K_{2}-B K_{1}\right], \\
R_{1}=R_{1}-\frac{R_{i}}{C} E \quad, \quad K_{2}=R_{2}-\frac{R_{i}}{C} F \\
C_{13}=\frac{f_{2} g_{1}}{m_{i} F} S_{9},
\end{gathered}
$$

The square of the $\mathcal{M}_{M M}+\delta \mathcal{M}$ amplitude gives a contribution to $M_{B R}$ of order $\left(\Delta m / m_{i}\right)^{2}$, therefore we neglect it in our approximation.

In the case of neutron decay the terms of order $\Delta m / m_{i}$ can also be neglected. Therefore $\mathcal{M}_{B R}$ can be approximated by the dominant $\tilde{\mathcal{M}}$ amplitude, and only the terms $C_{1}, C_{2}$ and $C_{3}$ are relevant for the $M_{B R}$ calculation. Neglecting the weak magnetism terms and 
the $q^{2}$ dependence of the form factors, and using the $p_{i} \approx p_{f}$ approximation, we get the bremsstrahlung matrix element squared for unpolarized neutron decay:

$$
\begin{gathered}
M_{B R}[n \rightarrow p e \bar{\nu} \gamma]=-32 G^{2} f_{1}^{2} e^{2} m_{i}^{2}\left[\left(1+\eta^{2}\right) H_{A}+\left(1-\eta^{2}\right) H_{B}\right], \\
H_{A}=2 E_{1}\left[E_{2} P^{2}+\frac{1}{K}-2 \frac{E_{2}}{F}-\frac{K}{F}+K \frac{m_{2}^{2}}{F^{2}}\right], \\
H_{B}=-D P^{2}+\frac{D+E}{F}+\frac{E_{2} E}{K F}-\frac{E_{1}}{K}-E \frac{m_{2}^{2}}{F^{2}}, \\
E_{1}=Q_{0}-K \quad, \quad P^{2}=\frac{1}{K^{2}}+\frac{m_{2}^{2}}{F^{2}}-2 \frac{E_{2}}{K F} \quad, \quad \eta=\frac{g_{1}}{f_{1}} .
\end{gathered}
$$

This expression is in complete agreement with the formulae of the app. in ref. [43].

\section{Model independent virtual correction}

The separation of the virtual amplitude into model dependent and model independent terms is explained in refs. [7,12,16] ( this separation was introduced by Sirlin in ref. [21] ). The model independent part of the virtual correction together with the photon bremsstrahlung defines the order- $\alpha$ model independent radiative correction. In the case of hyperon semileptonic decays, where $m_{i}-m_{f}>m_{2}$ and $E_{2}>>m_{2}$, the model independent virtual amplitude is approximately proportional to the zeroth-order amplitude ( see app. B in ref. [18]). The model independent virtual correction formulae are presented in refs. [14,16,18]. The result of the (2.19) soft photon integral can be found in app. A. From these results one can calculate the $W_{0 V S}\left(E_{2}, E_{f} ; z\right)$ function ( see eq. (2.26)). For the $E_{2} \gg m_{2}$ hyperon semileptonic decay events it is approximately proportional to the zeroth-order Dalitz-distribution:

$$
\begin{gathered}
W_{0 V S}\left(E_{2}, E_{f} ; z\right) \approx\left(1+Z_{V S}\right) W_{0}\left(E_{2}, E_{f}\right), \\
W_{0}\left(E_{2}, E_{f}\right)=\frac{G^{2}}{4 m_{i} \pi^{3}} L_{0}
\end{gathered}
$$

( see sect. 4 ). For charged hyperon decays:

$$
\begin{gathered}
Z_{V S}=\frac{\alpha}{\pi}\left\{\frac{3}{2} \ln \left(\frac{m_{i}}{m_{2}}\right)+2(S-1) \ln \left(\frac{2 \omega\left(E_{2}\right)}{m_{2}}\right)+2 S-2 S^{2}-\right. \\
\left.-\frac{3}{8}-\frac{\pi^{2}}{3}-2 \frac{E_{2}}{m_{i}}\left[\ln \left(\frac{2 E_{2}}{m_{i}}\right)-1\right]\right\}, \\
S=\ln \left(\frac{2 E_{2}}{m_{2}}\right) .
\end{gathered}
$$

For neutral hyperon decays :

$$
Z_{V S}=\frac{\alpha}{\pi}\left\{\frac{3}{2} \ln \left(\frac{m_{f}}{m_{2}}\right)+2(\hat{S}-1) \ln \left(\frac{2 \omega\left(E_{2}\right)}{m_{2}}\right)+S+\hat{S}-S^{2}-\hat{S}^{2}-\right.
$$




$$
\left.-\frac{3}{8}-\frac{\pi^{2}}{3}+2 \frac{\hat{E}_{2}}{m_{f}}\left[\ln \left(\frac{2 \hat{E}_{2}}{m_{f}}\right)-1\right]-2 \frac{\hat{E}_{2}-E_{2}}{E_{2}}\right\}+Z_{C b}
$$

where $Z_{C b}=\alpha \pi$ is the Coulomb correction, and

$$
\hat{E}_{2}=\frac{\left(p_{2} \cdot p_{f}\right)}{m_{f}}=\frac{Z}{m_{f}} \quad, \quad \hat{S}=\ln \left(\frac{2 \hat{E}_{2}}{m_{2}}\right) .
$$

In the case of neutron decay the $r_{V S}(x, y, \omega) / 100$ expression in the appendix of ref. [43] is a sufficient approximation for $Z_{V S}$ ( the $E_{2}>>m_{2}$ approximation has been employed in the calculation of eqs. $(5.3,5.4)$, therefore eq. (5.4) is not valid for neutron decay).

\section{Numerical results}

We have made several numerical calculations in order to check the Monte Carlo method described in sect. 2. First, we have calculated the model independent radiative correction to the neutron decay rate, using the (4.52) matrix element squared, and eq. (A2) in ref. [43]. We present below the results of program runs with two different $z$ and $N_{H}$ input values $\left(N_{H}\right.$ is the number of hard events that were generated ):

a, $z=0.01, \quad N_{H}=10^{4}$

$$
\begin{array}{cc}
r_{H}(z)=0.564 \quad, \quad \delta r_{H}(z)=0.004 \\
r_{\rho}=1.50 \quad, \quad r_{\rho}^{a n}=1.51 \\
E_{0 V S}=75.2 \quad, \quad E_{H}=38.4 \quad ;
\end{array}
$$

b, $\underline{z=0.001, \quad N_{H}=5 \cdot 10^{4}}$

$$
\begin{array}{cc}
r_{H}(z)=0.903 \quad, \quad \delta r_{H}(z)=0.003 \\
r_{\rho}=1.50 \quad, \quad r_{\rho}^{a n}=1.51 \\
E_{0 V S}=75.0 \quad, \quad E_{H}=39.8
\end{array}
$$

$r_{H}(z), E_{0 V S}$ and $E_{H}$ were defined in sect. 2 (eqs. $(2.69,2.63,2.64)$ ). $\delta r_{H}(z)$ is the statistical error of $r_{H}(z)$, calculated by eq. (2.60). $r_{\rho}$ is the Monte Carlo result for the relative radiative correction of the total decay rate:

$$
r_{\rho}=100 \frac{\rho-\rho_{0}}{\rho_{0}}
$$

and $r_{\rho}^{a n}$ is the same correction, calculated by the Sirlin-function ( see refs. [21,43]). The Coulomb correction is not included here. Eqs. $(6.1,6.2)$ show us that the $\delta r_{H}(z) / r_{H}(z)$ ratio is about $1 / \sqrt{N_{H}}$, and the $E_{H}$ efficiency of the hard bremsstrahlung generator is rather high.

The neutron decay bremsstrahlung has no collinear peak, because $m_{2}$ is not much smaller than $m_{i}-m_{f}$ ( see eq. (2.28) ). We can, however, put a smaller $m_{2}$ value into our Monte 
Carlo program, in order to see the effect of the collinear peak. Our method gives the following results for a small $m_{2}$ value:

$$
\begin{gathered}
m_{2}=0.01 \mathrm{MeV}, \quad z=0.001 \quad, \quad N_{H}=2 \cdot 10^{5} \\
r_{H}(z)=10.99 \quad, \quad \delta r_{H}(z)=0.02, \\
r_{\rho}=1.46 \quad, \quad r_{\rho}^{a n}=1.46 \quad, \\
E_{0 V S}=72.7 \quad, \quad E_{H}=36.0 .
\end{gathered}
$$

We have also calculated the radiative correction to the decay rate of the $\mu^{-} \rightarrow e \bar{\nu}_{e} \nu_{\mu}$, $\Sigma^{-} \rightarrow n e \bar{\nu}$ and $\Lambda \rightarrow p e \bar{\nu}$ decays ( see table 1 ). In the case of muon decay the virtual correction results of ref. [44] were employed, and the bremsstrahlung matrix element squared was precisely calculated by REDUCE. The $r_{\rho}^{a n}$ value presented in table 1 is the well-known analytical result of refs. [45,46]. For the hyperon decays the results of sections 4 and 5 were used.

The numerical results of eqs. $(6.1,6.2,6.4)$ and table 1 show us that

$$
\delta \rho_{H}(z) / \rho_{H}(z) \sim \frac{1}{\sqrt{N_{H}}},
$$

and

$$
\frac{E_{H}}{E_{0 V S}} \sim \frac{1}{2}
$$

Therefore our Monte Carlo method is very efficient. The radiative correction calculations by weighted event generations do not require much computer time, due to eq. (6.5), and the high $E_{0 V S}, E_{H}$ efficiency values make this method useful for unweighted event generation.

The $g$ approximate function ( see eq. (2.41) ) removes completely the infrared peak and the electron-photon collinear peak from $M_{B R}$. Therefore, we expect our Monte Carlo method to be efficient for all charged semileptonic decays, and for those neutral semileptonic decays where the outgoing charged particle is not ultrarelativistic. In order to see the Monte Carlo efficiency dependence on the outgoing charged particle mass, we have computed the $E_{H}$ efficiency in $\Lambda \rightarrow p e \bar{\nu}$ decay for various proton mass values ( see table 2 ). For the $m_{f}<<m_{i}$ neutral semileptonic decays one could employ the "channel method", described in ref. [37], in order to get higher $E_{H}$ efficiencies.

We have calculated also various bin distributions ( see eqs. (2.66-2.71)). The radiative correction to the electron energy bin distribution was computed in the case of the neutron and muon decays, and the results were compared to the bin distribution corrections obtained by using the analytical correction functions of refs. [21] and [46]. We have also alculated the photon energy bin distribution for muon decay, and we compared it to the analytical result of refs. [47,48]. Complete agreement was found for each bin distribution. In the case of hyperon semileptonic decays we calculated the $E_{2}, E_{f}$ and $\cos \theta_{e \nu}$ bin distribution corrections ( $\theta_{e \nu}$ is defined as the angle between the $\underline{p_{2}}$ and the $-\left(\underline{p_{2}}+p_{f}\right)$ vectors ). Using the same theoretical framework and input parameters as in ref. [16], we were able to compare our Monte Carlo results to the numerical tables of ref. [16]. For this purpose, we have applied cubic line interpolation and extrapolation to the results of tables 3, 5 and 6 in [16]. Here, also, complete agreement between the two calculations was found. 
We have made a detailed numerical investigation in order to see the contributions of the $C_{j}(j=1, \ldots, 13)$ terms of the bremsstrahlung matrix element squared to the various measurable quantities. One can judge the order of magnitude of the $C_{j}$ terms from the results presented in table 3 . Here we vave calculated the model independent relative radiative correction to the decay rate of $0.1 E_{2 m}<E_{2}<0.4 E_{2 m}$ events ( $E_{2 m}$ is the electron energy maximum, see eq. (2.10) ). The numbers in the $1,1-3$ and $1-13$ columns show complete (virtual+bremsstrahlung ) radiative corrections ( $1: M_{B R}=C_{1} ; 1-3: M_{B R}=C_{1}+C_{2}+C_{3}$, etc.). The other columns contain only the hard bremsstrahlung correction. It is remarkable that the contributions of the $C_{4}$ through $C_{13}$ terms are very small. We have calculated the radiative correction to the $E_{2}$ and $\cos \theta_{e \nu}$ bin distributions for $\Sigma^{-} \rightarrow n e \bar{\nu}$ and $\Lambda \rightarrow p e \bar{\nu}$ decays, first with the $M_{B R}=C_{1}+\ldots+C_{13}$ complete bremsstrahlung, and second with the $M_{B R}=C_{1}+C_{2}+C_{3}$ truncated bremsstrahlung. These two distributions differ by less than $0.1 \%$, implying that the $C_{4}$ through $C_{13}$ terms have very small effect on the unpolarized distributions.

We have calculated the relative radiative corrections to the $E_{2}$ and $\cos \theta_{e \nu}$ bin distributions with $\lambda_{f}=\lambda_{g}=0$, that is neglecting the $q^{2}$ dependence of the form factors both in the zeroth-order and in the radiatively corrected distributions ( see sect. 3 ). We have compared these results with those corrections where the $q^{2}$ dependence of the form factors was properly taken into account. These two corrections differ by less than $0.1 \%$. This result suggests that, in general, the relative radiative corrections are not sensitive to the $q^{2}$ dependence of the form factors. In ref. [16] relative radiative corrections were tabulated for several distributions of unpolarized hyperon decays $\left(\Sigma^{-} \rightarrow n e \bar{\nu}, \Sigma^{-} \rightarrow \Lambda e \bar{\nu}, \Xi^{-} \rightarrow \Lambda e \bar{\nu}, \Lambda \rightarrow p e \bar{\nu}\right.$ ), using $\lambda_{f}=\lambda_{g}=0$ for both the zeroth-order distributions and for the radiative corrections. The relative correction results published in this paper should be, therefore, multiplied by the zeroth-order distributions expressed by $q^{2}$ dependent form factors, in order to get precise radiatively corrected distributions.

We have published in ref. [49] relative radiative correction results for several bin distributions of $\Lambda \rightarrow p e \bar{\nu}$ decay. These corrections were calculated by the Monte Carlo method described in ref. [29]. We have repeated these calculations, by using our new Monte Carlo method. Good agreement between the two computations was found. The computer time needed to achieve a given statistical accuracy by the new method was 10 times smaller than by the method of ref. [29]. The results of ref. [49] illustrate the importance of the reliable and precise hard photon calculations for the experimental analyses. 


\section{Acknowledgements}

This work was supported by the Hungarian Soros Foundation, by CERN Theor. Div. and by OTKA F4011 of the Hungarian Academy of Sciences. One of the authors ( F. G. ) would like to thank Prof. John Ellis for the possibility of a two months visit to CERN TH Division where the main part of this work was done. He also thanks Dr. Kálmán Tóth for very useful discussions.

\section{Appendix A}

In the case of charged semileptonic decays $\left(p=p_{i}\right)$ the (2.19) integral can be exactly calculated, without any approximation [46] :

$$
Z_{S}[\text { charged }]=\frac{\alpha}{\pi}\left\{2\left(\frac{S}{\beta}-1\right) \ln \left(\frac{2 \hat{\omega}}{\lambda}\right)+\frac{1}{\beta} L\left(\frac{2 \beta}{1+\beta}\right)+\frac{S}{\beta}-\frac{S^{2}}{\beta}+1\right\},
$$

where

$$
\beta=\frac{\left|\underline{p}_{2}\right|}{E_{2}} \quad, \quad S=\frac{1}{2} \ln \left(\frac{1+\beta}{1-\beta}\right),
$$

and $L$ is the Spence function :

$$
L(x)=\int_{0}^{x} d t \frac{\ln |1-t|}{t}
$$

Eq. (A1) is also valid for neutron decay, where the terms of order $\Delta m / m_{i}$ can be neglected $\left(\Delta m=m_{i}-m_{f}\right)$.

In the case of neutral semileptonic decays eq. (7.3) of ref. [50] has been employed. For the semileptonic decays of the $\mathrm{SU}(3)$-octet baryons the $\Delta m / m_{i} \ll 1$ and $\beta \approx 1$ properties are useful in order to get a simple expression :

$$
\begin{aligned}
Z_{S}\left[\text { neutral, } \Delta m / m_{i}\right. & \ll 1, \beta \approx 1] \approx \frac{\alpha}{\pi}\left\{2(\hat{S}-1) \ln \left(\frac{2 \hat{\omega}}{\lambda}\right)+S-S^{2}-\right. \\
& \left.-\frac{\pi^{2}}{6}+1-2 \frac{\hat{E}_{2}-E_{2}}{E_{2}}\right\},
\end{aligned}
$$

where

$$
\hat{S}=\ln \left(\frac{2 \hat{E}_{2}}{m_{2}}\right) \quad, \quad \hat{E}_{2}=\frac{\left(p_{2} \cdot p_{f}\right)}{m_{f}}=\frac{Z}{m_{f}}
$$

( $Z$ is given in app. B ).

Eq. (A4) is valid up to terms of order $\Delta m / m_{i}$ ( the neglected terms are of order $\left.\left(\Delta m / m_{i}\right)^{2}\right)$. 


\section{Appendix B}

We employ the letters $A, B, C, D, E, F, X, Y, Z$ and $W$ to denote the 10 scalar products of the $p_{i}, p_{1}, p_{2}, k$ and $p_{f}$ four-momenta. These scalar products can be expressed by the $M^{2}, E_{2}, K, \cos \theta_{1}^{*}$ and $\phi_{1}^{*}$ Monte Carlo variables and the $m_{i}, m_{f}, m_{2}$ masses in the following manner :

$$
\begin{gathered}
A:=\left(p_{i} \cdot p_{1}\right)=m_{i}\left(Q_{0}-K\right), \\
B:=\left(p_{i} \cdot p_{2}\right)=m_{i} E_{2}, \\
C:=\left(p_{i} \cdot k\right)=m_{i} K, \\
D:=\left(p_{1} \cdot p_{2}\right)=N_{12}-E-F, \\
E:=\left(p_{1} \cdot k\right)=\left(Q_{0}^{2}-Q^{2}\right) / 2, \\
F:=\left(p_{2} \cdot k\right)=m_{i}\left(K+E_{2}-E_{0}\right), \\
X:=\left(p_{i} \cdot p_{f}\right)=m_{i} E_{f}, \\
Y:=\left(p_{1} \cdot p_{f}\right)=A-D-E, \\
Z:=\left(p_{2} \cdot p_{f}\right)=B-D-F-m_{2}^{2}, \\
W:=\left(p_{f} \cdot k\right)=C-E-F,
\end{gathered}
$$

$N_{12}$ is equal to the $\left(p_{1} \cdot p_{2}\right)$ scalar product in zeroth-order ( if $\left.K=0\right)$ :

$$
N_{12}=m_{i}\left(E_{f m}-E_{f}\right)
$$

$E_{f m}, Q_{0}$ and $E_{0}$ are expressed in eqs. (2.7), (2.14) and (2.33). $E_{f}$ and $Q=\left|\underline{p}_{2}+\underline{p}_{f}\right|=\left|\underline{p}_{1}+\underline{k}\right|$ can be calculated by the following equations:

$$
\begin{gathered}
E_{2 K}=E_{2}+K \quad, \quad P=\sqrt{m_{i}^{2}-M^{2}+E_{2 K}^{2}-2 m_{i} E_{2 K}}, \\
\gamma_{M}=\frac{m_{i}-E_{2 K}}{M} \quad, \quad \beta_{M}=\frac{P}{m_{i}-E_{2 K}}, \\
E_{1}^{*}=\frac{M^{2}-m_{f}^{2}}{2 M}, \quad E_{1}=\gamma_{M} E_{1}^{*}\left(1+\beta_{M} \cos \theta_{1}^{*}\right), \\
E_{f}=m_{i}-E_{2}-K-E_{1}, \\
k_{z}=\frac{m_{i}\left(E_{M}-E_{2}\right)-K\left(m_{i}-E_{2}-K\right)}{P}, \\
p_{1 z}=-\gamma_{M} E_{1}^{*}\left(\cos \theta_{1}^{*}+\beta_{M}\right), \\
p_{1 x}=E_{1}^{*} \sin \theta_{1}^{*} \cos \phi_{1}^{*}, \quad p_{1 y}=E_{1}^{*} \sin \theta_{1}^{*} \sin \phi_{1}^{*}, \\
k_{x}=\sqrt{K^{2}-k_{z}^{2}}, \\
Q=k_{x}+p_{1 x} \quad, \quad Q_{y}=p_{1 y} \quad, \quad Q_{z}=k_{z}+p_{1 z}
\end{gathered}
$$




\section{References}

1. J. Wise et al., Phys. Lett. 91B (1980) 165;

98B (1981) 123;

D. Jensen et al., Proc. Int. Conf. High-Energy Phys., Brighton, 1983, Rutherford

Appleton Lab. (ed.: J. Guy and C. Costain ), p. 255.

2. M. Bourquin et al., Z. Phys. C12 (1982) 307;

C21 (1983) 1, 17, 27.

3. S. Y. Hsueh et al., Phys. Rev. D38 (1988) 2056.

4. J. Dworkin et al., Phys. Rev. D41 (1990) 780.

5. S. Suzuki and Y. Yokoo, Nucl. Phys. B94 (1975) 431.

6. K. Fujikawa and M. Igarashi, Nucl. Phys. B103 (1976) 497.

7. A. Garcia and S. R. Juárez W., Phys. Rev. D22 (1980) 1132;

D22 (1980) 2923(E).

8. A. Baltas, K. Gavroglou and D. Photinos, Nuovo Cimento 66A (1981) 399.

9. A. Garcia, Phys. Rev. D25 (1982) 1348.

10. A. Garcia, Phys. Rev. D28 (1983) 1659.

11. A. Garcia and S. R. Juárez W., Phys. Rev. D28 (1983) 2180.

12. K. Tóth, K. Szegö and A. Margaritis, Phys. Rev. D33 (1986) 3306.

13. S. R. Juárez W., A. Martinez V. and A. Garcia, Phys. Rev. D35 (1987) 232;

Phys. Rev. D38 (1988) 2904;

Phys. Rev. D43 (1991) 282.

14. K. Tóth anf F. Glück, Phys. Rev. D40 (1989) 119.

15. D. M. Tun, S. R. Juárez W. and A. Garcia, Phys. Rev. D40 (1989) 2967.

16. F. Glück and K. Tóth, Phys. Rev. D41 (1990) 2160.

17. D. M. Tun, S. R. Juárez W. and A. Garcia, Phys. Rev. D44 (1991) 3589.

18. F. Glück and K. Tóth, Phys. Rev. D46 (1992) 2090.

19. A. Martinez, A. Garcia and D. M. Tun, Phys. Rev. D47 (1993) 3984.

20. S. R. Juárez W., Phys. Rev. D48 (1993) 5233.

21. A. Sirlin, Phys. Rev. 164 (1967) 1767.

22. F. E. Low, Phys. Rev. 110 (1958) 974.

23. H. Chew, Phys. Rev. 123 (1961) 377.

24. S. L. Adler and Y. Dothan, Phys. Rev. 151 (1966) 1267.

25. J. Pestiaeu, Phys. Rev. 160 (1967) 1555.

26. E. M. Nyman, Phys. Rev. 170 (1968) 1628.

27. E. Fischbach and J. Smith, Phys. Rev. 184 (1969) 1645.

28. K. Tóth, private communication.

29. F. Glück and I. Joó, KFKI-1993-18/A ( unpublished ).

30. R. Hagedorn, Relativistic Kinematics (1964), W. A. Benjamin, Inc., New-York.

31. J. H. Friedman, J. Comp. Phys. 7 (1971) 201.

32. E. Byckling and K. Kajantie, Particle Kinematics (1973), J.Wiley and Sons, New-York.

33. F. James, Rep. Prog. Phys. 43 (1980) 1145.

34. F. A. Berends and R. Kleiss, Nucl. Phys. 177 (1981) 237.

35. F. A. Berends, R. Kleiss and S. Jadach, Comp. Phys. Comm. 29 (1983) 185.

36. V. Barger and R. Phillips, Collider Physics (1987), Addison-Wesley Publ. Comp.

37. F. A. Berends, R. Kleiss and W. Hollik, Nucl. Phys. B304 (1988) 712.

38. R. Kleiss, in Z physics at Lep 1, CERN-89-08 (1989) Vol 3., p. 1, ed.: G. Altarelli,

R. Kleiss and C. Verzegnassi.

39. R. Kleiss, CERN-TH.5732/90 (1990).

40. M. Gaillard and G. Sauvage, Ann. Rev. Nucl. Part. Sci. 34 (1985) 351.

41. T. H. Burnett and N. M. Kroll, Phys. Rev. Lett. 20 (1968) 86.

42. H. W. Fearing, Phys. Rev. C6 (1972) 1136.

43. F. Glück, Phys. Rev. D47 (1993) 2840.

44. R. E. Behrends, R. J. Finkelstein and A. Sirlin, Phys. Rev. 101 (1956) 866.

45. S. M. Berman, Phys. Rev. 112 (1958) 267.

46. T. Kinoshita and A. Sirlin, Phys. Rev. 113 (1959) 1652.

47. T. Kinoshita and A. Sirlin, Phys. Rev. Lett. 2 (1959) 177.

48. S. G. Eckstein and R. H. Pratt, Ann. of Phys. 8 (1959) 297.

49. F. Glück and I. Joó, KFKI-1993-22/A ( submitted to Phys. Rev. D ).

50. G.'t Hooft and M. Veltman, Nucl. Phys. B153 (1979) 365. 
Table 1.

Monte Carlo results for $\mu^{-} \rightarrow e \bar{\nu}_{e} \nu_{\mu}, \Sigma^{-} \rightarrow n e \bar{\nu}$ and $\Lambda \rightarrow p e \bar{\nu}$ decays ( $z=0.001 ; N_{H}=10^{6}$ for muon decay, $N_{H}=10^{5}$ for the others )

$\begin{array}{lccc} & \mu^{-} \rightarrow e \bar{\nu}_{e} \nu_{\mu} & \Sigma^{-} \rightarrow n e \bar{\nu} & \Lambda \rightarrow p e \bar{\nu} \\ r_{H}(z) & 13.51 & 15.31 & 14.36 \\ \delta r_{H}(z) & 0.01 & 0.04 & 0.04 \\ r_{\rho} & -0.43 & -0.10 & 1.86 \\ r_{\rho}^{a n} & -0.42 & - & - \\ E_{0 V S} & 63.5 & 52.7 & 71.0 \\ E_{H} & 30.2 & 28.0 & 38.0\end{array}$

Table 2.

$E_{H}$ efficiencies in $\Lambda \rightarrow p e \bar{\nu}$ decay for various proton masses

$\begin{array}{lccccccc}m_{p}(\mathrm{GeV}) & 0.94 & 0.5 & 0.4 & 0.3 & 0.2 & 0.1 & 0.05 \\ E_{H} & 38 & 24 & 18 & 9.6 & 4.5 & 1.2 & 0.4\end{array}$

Table 3.

Contributions of the $C_{j}$ terms to the radiative correction for $0.1 E_{2 m}<E_{2}<0.4 E_{2 m}$ events ( $z=0.001$ )

$\begin{array}{lccccccc}C_{j} \text { terms } & 1 & 1-3 & 1-13 & 4-5 & 6-9 & 10-11 & 12-13 \\ \Sigma^{-} \rightarrow n e \bar{\nu} & 1.13 & 4.53 & 4.50 & -0.04 & 0.01 & -0.02 & 0.02 \\ \Lambda \rightarrow p e \bar{\nu} & 3.27 & 6.50 & 6.57 & 0.04 & 0.02 & -0.01 & 0.02\end{array}$

